Pacific Journal of Mathematics

ON THE ELEMENTARY RENEWAL THEOREM FOR 


\title{
ON THE ELEMENTARY RENEWAL THEOREM FOR NON-IDENTICALLY DISTRIBUTED VARIABLES
}

\author{
WALTER L. SMith
}

1. Introduction. Let $\left\{X_{n}\right\}$ be a sequence of independent, identically distributed random variables with $0<E X_{n}<\infty$; write $\mathrm{S}_{n}=$ $X_{1}+X_{2}+\cdots+X_{n}$; let $N_{x}$ be the number of partial sums $S_{n} \leq x$; write $H(x)=E N_{x}$. The Elementary Renewal Theorem states that under certain conditions $H(x) / x \rightarrow\left\{E X_{n}\right\}^{-1}$ as $x \rightarrow \infty$.

Kawata (1956) has proved a result which, as we shall see below, is equivalent to a generalization of the Elementary Renewal Theorem to the case in which the $\left\{X_{n}\right\}$ are non-identically distributed. Unfortunately, he found it necessary to impose quite heavy restrictions upon the distribution functions involved. In this note we shall also be concerned with the proof of the Elementary Renewal Theorem for non-identically distributed random variables, but under substantially weaker conditions than Kawata's. This renewal theorem, essentially, provides an asymptotic estimate to the sum $\sum_{n=1}^{\infty} P\left\{S_{n} \leqq x\right\}$; actually, we shall discuss in this paper the asymptotic behavior of more general sums $\sum_{n=1}^{\infty} a_{n} P\left\{S_{n} \leqq x\right\}$, for certain general classes of positive coefficient-sequences $\left\{a_{n}\right\}$. Such more general sums have also been considered by Hatori (1959), (1960), who followed Kawata's general line of attack, however, and was consequently led to assume unduly restrictive conditions.

It is well if we point out that there is another line of inquiry which could be pursued in the present context, one with which the present investigation must not be confused. Instead of considering $N_{x}$, one could define a random variable $M_{x}$ as the least $m$ for which $S_{m}>x$, and then study the asymptotic behavior of $E M_{x} / x$. The latter problem (also for non-identically distributed $\left\{X_{n}\right\}$ ) has been tackled in recent work announced by Robbins and Chow (1962)*. However, as might be expected, the problem we consider and the problem considered by Robbins and Chow differ in important respects, in general. Indeed, a reference to Theorem A, which we quote below, will show that one can construct a sequence of independent and identically distributed random variables with a finite first moment, for which $E M_{x}$ is finite but $E N_{x}$ is infinite. Evidently conditions which are

Received June 7, 1962, and in revised form March 7, 1963.

This research was supported by the Office of Naval Research under contract No. Ncnr-855 (09) for research in probability and statistics at the University of North Carolina, Chapel Hill, N. C. Reproduction in whole or in part is permitted for any purpose of the United States Government.

* Footnote added in proof:-The details of this work have now appeared in Ann. Math. Statist., 34 (1963), 390-395. 
adequate for a study of $M_{x}$ may prove inadequate for a similar study of $N_{x}$. However, when all the $\left\{X_{n}\right\}$ are nonnegative we have an exceptional case; for then $M_{x}=N_{x}+1$, and the distinction between the two lines of inquiry disappears. Our main result, Theorem 1 announced below, is more general than the one announced by Robbins and Chow, for the case of nonnegative random variables.

Let us write $F_{n}(x) \equiv P\left\{X_{n} \leqq x\right\}$ and $G_{n}(x) \equiv P\left\{S_{n} \leqq x\right\}$; we shall also need the unit function $U(x) \equiv P\{0 \leqq x\}$.

The function $L(x)$, defined for all sufficiently large $x$, is said to be a function of slow growth if, for every $c>0$

$$
\frac{L(c x)}{L(x)} \rightarrow 1, \quad \text { as } x \rightarrow \infty \text {. }
$$

It follows from the work of Karamata (1930), that a nonnegative function of slow growth can always be represented thus:

$$
L(x)=\frac{a(x)}{x} \exp \left\{\int_{1}^{x} \frac{a(u)}{u} d u\right\},
$$

where $a(x)$ is a function which tends to unity as $x$ tends to infinity. An easy consequence of this representation (1.2) is that the convergence (1.1) takes place uniformly with respect to $c$ in any interval not containing the origin.

As a final preliminary we must say a word about the non-negative coefficient-sequences $\left\{a_{n}\right\}$ which we consider. For such a sequence we shall suppose there exist numbers $\alpha>0, \gamma \geqq 0$, and some nonnegative function of slow growth $L(x)$, such that

$$
\sum_{n=1}^{\infty} a_{n} x^{n} \sim \frac{\alpha}{(1-x)^{\gamma}} L\left(\frac{1}{1-x}\right), \quad \text { as } x \rightarrow 1-0 .
$$

By an appeal to a Tauberian theorem due to Karamata (Hardy, 1949, p. 166) it is possible to deduce from (1.3) that

$$
\sum_{n=1}^{N} a_{n} \sim \frac{\alpha N^{\gamma} L(N)}{\Gamma(1+\gamma)}, \quad \text { as } N \rightarrow \infty,
$$

although we shall omit details of this deduction. Conversely, if one starts from (1.4) then an appropriate Abelian theorem will show that (1.3) follows. Thus (1.3) and (1.4) are equivalent assumptions on the nonnegative $\left\{a_{n}\right\}$. We also note, as an easy deduction from (1.4), that

$$
a_{n}=o\left(n^{\gamma} L(n)\right), \quad \text { as } n \rightarrow \infty .
$$

In connection with these sequences $\left\{a_{n}\right\}$ we need to define an index: 
Definition. An index $k$ of the sequence $\left\{a_{n}\right\}$ is any number $k$ such that $a_{n}=O\left(n^{k}\right)$.

If we write $k^{*}$ for the greatest lower bound of the indexes of the sequence $\left\{a_{n}\right\}$ then $k^{*}$ may or may not be an index itself. From (1.5) it is clear that $k^{*} \leqq \gamma$. On the other hand, we can infer from (1.4) that $k^{*} \geqq \gamma-1$ and that $\gamma-1$ can only be an index if $L(n)$ is a bounded function. We have, therefore,

Lemma 1. If $k^{*}$ is the greatest lower bound of the indexes of $\left\{a_{n}\right\}$ then $\gamma-1 \leqq k^{*} \leqq \gamma$; the number $\gamma-1$ cannot be an index unless $L(n)$ is a bounded function.

The main result of this paper can now be stated.

THEOREM 1. Suppose the following conditions hold.

(T1) $\left\{X_{n}\right\}$ is a sequence of independent random variables with distribution functions $\left\{F_{n}(x)\right\}$ and finite expectations $\mu_{n}=E X_{n}$, such that

$$
\frac{\mu_{1}+\mu_{2}+\cdots+\mu_{n}}{n} \rightarrow \mu, \quad \text { as } n \rightarrow \infty,
$$

where $\mu$ finite and strictly positive.

(T2) For every $\varepsilon>0$

$$
\int_{n \varepsilon}^{\infty} \frac{1}{n} \sum_{r=1}^{n}\left\{1-F_{r}(x)\right\} d x \rightarrow 0, \quad \text { as } n \rightarrow \infty .
$$

(T3) For some $\alpha>0, \gamma \geqq 0$, and some nonnegative function of slow growth $L(x)$, the sequence of nonnegative constants $\left\{a_{n}\right\}$ satisfies either of the equivalent asymptotic relations (1.3) or (1.4).

(T4) $\sum_{n=1}^{\infty} a_{n}$ diverges.

Then, if we write $G_{n}(x)=P\left\{X_{1}+X_{2}+\cdots+X_{n} \leqq x\right\}$, in order that

$$
\sum_{n=1}^{\infty} a_{n} G_{n}(x) \sim \frac{\alpha L(x)}{\Gamma(1+\gamma)}\left(\frac{x}{\mu}\right)^{\gamma}, \quad \text { as } x \rightarrow \infty,
$$

it is sufficient that one of the following two sets of conditions, (T5) or (T6), hold.

(T5) The $X_{n}$ are nonnegative, in which case it will be proved that there necessarily exists some unbounded non-decreasing function $l(n)$ such that

$$
\lim _{n \rightarrow \infty} \frac{1}{n} \sum_{r=1}^{n} \int_{0}^{r / l(r)}\left\{1-F_{r}(x)\right\} d x=\mu .
$$


(T6) (a) If $k$ is an index of $\left\{a_{n}\right\}$, then there is a distribution function $K(x)$ of a negative-valued random variable with a finite moment of order $(k+2)$, such that $K(x) \geqq F_{n}(x)$ for all $n$ and all $x$; (b) If $-\kappa$ is the first moment of $K(x)$, then for some $v>\kappa$ and every $\varepsilon>0$,

$$
\lim _{n \rightarrow \infty} \inf \int_{-\infty}^{\varepsilon n / \log n} \frac{1}{n} \sum_{r=1}^{n}\left\{U(x)-F_{r}(x)\right\} d x>2 \sqrt{(k+1) v \varepsilon} .
$$

In condition (T4) above we have required that $\sum_{n=1}^{\infty} a_{n}$ shall diverge; it will be appreciated that this assumption is made only to avoid triviality. A consequence of the divergence of $\sum_{i=1}^{\infty} a_{n}$ is that the index $k \geqq-1$; therefore the distribution function $K(x)$ which appears in condition (T6)(a) will always have a finite mean; this justifies the introduction of $-\kappa$ in condition $(\mathrm{T} 6)(\mathrm{b})$.

The special case of Theorem 1 in which the $\left\{X_{n}\right\}$ are nonnegative can be given the following form.

THEOREM 2. Suppose that (i) $\left\{X_{n}\right\}$ is a sequence of nonnegative random variables such that (T1) and (T2) of Theorem 1 hold; (ii) $A(n)$ is a non-decreasing function of $n$ for which constants $\alpha>0$, $\gamma \geqq 0$, and a nonnegative function of slow growth $L(n)$, can be found such that

$$
A(n) \sim \alpha n^{\gamma} L(n), \quad \text { as } n \rightarrow \infty .
$$

Then it follows that

$$
E A\left(N_{x}\right) \sim \alpha\left(\frac{x}{\mu}\right)^{\gamma} L(x), \quad x \rightarrow \infty .
$$

We note that by letting $A(n)=n$ in Theorem 2 we obtain a version of the Elementary Renewal Theorem for independent, nonidentically distributed, nonnegative random variables. Alternatively, by taking $a_{n}=1$ for all $n$ in Theorem 1, we obtain the following version for the case when the random variables may assume negative values.

THEOREM 3. If conditions (T1) and (T2) of Theorem 1 hold; and if both parts of (T6) hold for $k=0$, then

$$
\frac{H(x)}{x} \rightarrow \frac{1}{\mu}, \quad \text { as } x \rightarrow \infty,
$$

where $H(x)=\sum_{n=1}^{\infty} G_{n}(x)$ is the expected number of partial sums $S_{n} \leqq x$. 
From (1.13) we can infer that, for any fixed $h>$

$$
\frac{1}{t} \int_{t}^{t+h} H(x) d x \rightarrow \frac{h}{\mu}, \quad \text { as } t \rightarrow \infty
$$

Therefore

$$
\frac{1}{t} \int_{0}^{t}\{H(x+h)-H(x)\} d x \rightarrow \frac{h}{\mu}, \quad \text { as } t \rightarrow \infty
$$

or, in other words,

$$
\lim _{t \rightarrow \infty} \frac{1}{t} \int_{0}^{t} \sum_{n=1}^{\infty} P\left\{x<S_{n} \leqq x+h\right\} d x=\frac{h}{\mu} .
$$

This last limit (1.15) is the form taken by Kawata's result (1956); we see that it is implied by the simpler statement (1.13). On the other hand it is not difficult to deduce (1.13) from (1.15), so that (1.15) seems an unduly complicated form for the result. For (1.15) is equivalent to (1.14); and from (1.14) and the monotone character of $H(x)$ we can infer that $\lim \sup H(t) / t \leqq \mu^{-1}$ and $\lim$ inf $H(t+h) / t \geqq \mu^{-1}$.

We close this introduction with some remarks about the conditions of Theorem 1. The easiest proofs of the Elementary Renewal Theorem for the case of identically distributed random variables make use of the weak law of large numbers to show that $S_{n} / n \rightarrow \mu$ in probability, as $n \rightarrow \infty$. The present investigation will also depend on establishing such weak convergence of $S_{n} / n$, and conditions (T1) and (T2), aided by (T6)(a) when the random variables can take negative values, are concerned with this task.

To understand the raison d'être of condition (T6)(b) it is necessary to inquire a little into our mode of proof. We shall, as just noted, begin by establishing that $S_{n} / n \rightarrow \mu$ in probability. If only $S_{n}$ would not fluctuate too violently about its expected value $n \mu$ our theorems would then be an easy consequence of this weak law of large numbers. Unfortunately, considerable deviation of $S_{n}$ from $n \mu$ is possible; the main obstacle we have to overcome is presented by sequences $\left\{S_{n}\right\}$ which tend to decrease steadily over long stretches of $n$ and then indulge in a rare, but very large, increase in value. This kind of awkward behavior is exemplified by sequences $\left\{F_{n}(x)\right\}$ which assign nearly all the probability to the negative values of $x$ and reserve only a very small probability for positive values of $x$, necessarily located at very high positive values in order to make the expectations come right. Condition $(\mathrm{T} 6)(\mathrm{b})$ is concerned with controlling this kind of awkwardness. 
Condition (T6)(a), which is unnecessary when the random variables are nonnegative, is introduced to ensure the finiteness of the quantities with which we deal; it will be understood better in relation to the following theorem, which we shall use later in this paper but prove elsewhere (Smith (1964)).

THEOREM A.* If $\left\{X_{n}\right\}$ is a sequence of independent and identically distributed random variables with $0<E X_{n}<\infty$, and if $k \geqq 1$, then a necessary and sufficient condition for the convergence of the series

$$
\sum_{n=1}^{\infty} n^{k} P\left\{X_{1}+X_{2}+\cdots+X_{n} \leqq x\right\},-\infty<x<+\infty,
$$

is that $E\left\{\left|\min \left(0, X_{n}\right)\right|^{k+2}\right\}<\infty$. Furthermore, when this condition is met, if $X_{0}$ is any other random variable, independent of the $\left\{X_{n}\right\}$, such that $E\left\{\left|\min \left(0, X_{0}\right)\right|^{k+2}\right\}<\infty$, then

$$
\sum_{n=1}^{\infty} n^{k} P\left\{X_{0}+X_{1}+\cdots+X_{n} \leqq x\right\},-\infty<x<+\infty,
$$

is also convergent.

Thus we see that (T6)(a) must be satisfied when the $\left\{X_{n}\right\}$ are identically distributed, and it therefore seems reasonable to require the satisfaction of some condition like (T6)(a) even in the general case, if we are to ensure the finiteness of the left-hand side of (1.8).

2. Some preliminary lemmas. We begin by showing that the conditions of Theorem 1 are sufficient to ensure that $S_{n} / n \rightarrow \mu$ in probability as $n \rightarrow \infty$. This could be done by appeal to classical results; however, it is not difficult to proceed from first principles, and our proof conveniently introduces an argument of a sort which we shall use several times in the course of this paper.

Lemma 2. If conditions (T1) and (T2) hold, then a sufficient condition for ensuring that $S_{n} / n \rightarrow \mu$ in probability as $n \rightarrow \infty$ is either: (a) the random variables $\left\{X_{n}\right\}$ are nonnegative; or (b) condition (T6)(a) holds with $k=-1$.

Proof. Consider first the case of a sequence of independent, nonnegative, random variables $\left\{X_{n}\right\}$ whose distribution functions satisfy (T2) and whose mean values satisfy the condition that $\mu_{1}+\mu_{2}+\cdots+\mu_{n}=O(n)$, a condition less restrictive than (1.6). Write $m_{n}=n^{-1}\left(\mu_{1}+\mu_{2}+\cdots+\mu_{n}\right)$

* Footnote: This theorem is also a fairly easy deduction from the recently published results of M. L. Katz (1963). 
and let $\eta$ be a small strictly positive number. Then, in virtue of a familiar inequality, for every fixed $t \geqq 0$ we have

$$
P\left\{S_{n} \leqq n\left(m_{n}-\eta\right)\right\} \leqq e^{n\left(m_{n}-\eta\right) t} E\left\{e^{-t S_{n}}\right\} .
$$

Furthermore, if we make use of the Laplace-Stieltjes transforms

$$
\Phi_{j}(t)=\int_{0-}^{\infty} e^{-t x} d F_{j}(x),
$$

we may rewrite this last inequality thus:

$$
P\left\{S_{n} \leqq n\left(m_{n}-\eta\right)\right\} \leqq e^{n\left(m_{n}-\eta\right) t} \prod_{j=1}^{n} \Phi_{j}(t) .
$$

But, as may easily be verified,

$$
\Phi_{j}(t) \leqq e^{\Phi_{j}(t)-1} ;
$$

and so we have that

$$
P\left\{S_{n} \leqq n\left(m_{n}-\eta\right)\right\} \leqq e^{W} n^{(t)},
$$

where, after some integrations by parts, we see that

$$
W_{n}(t)=n\left(m_{n}-\eta\right) t-t \sum_{j=1}^{n} \int_{0}^{\infty} e^{-t x}\left\{1-F_{j}(x)\right\} d x .
$$

Choose a small $\varepsilon>0$ and set

$$
\delta_{n}(\varepsilon)=\int_{n \varepsilon}^{\infty} \frac{1}{n} \sum_{j=1}^{n}\left\{1-F_{j}(x)\right\} d x ;
$$

by (T2), $\delta_{n}(\varepsilon) \rightarrow 0$ as $n \rightarrow \infty$. From (2.2) we then deduce that

$$
W_{n}(t) \leqq n\left(m_{n}-\eta\right) t-n t e^{-n t \varepsilon}\left[\delta_{n}(0)-\delta_{n}(\varepsilon)\right] .
$$

If we observe that $\delta_{n}(0)=m_{n}$ and put $t=(n \sqrt{\varepsilon})^{-1}$ in (2.3), we find that

$$
\begin{aligned}
& W_{n}\left(\frac{1}{n \sqrt{\varepsilon}}\right) \leqq \frac{m_{n}-\eta}{\sqrt{\varepsilon}}-\frac{\exp (-\sqrt{\varepsilon})}{\sqrt{\varepsilon}}\left[m_{n}-\delta_{n}(\varepsilon)\right] \\
& \quad=\frac{1}{\sqrt{\varepsilon}}\left\{m_{n}[1-\exp (-\sqrt{\varepsilon})]+\exp (-\sqrt{\varepsilon}) \delta_{n}(\varepsilon)-\eta\right\} .
\end{aligned}
$$

Recall that $m_{n}$ is positive and bounded; thus $m_{n}[1-\exp (-\sqrt{\varepsilon})]$ can be made arbitrarily small for all $n$, by choosing $\varepsilon$ sufficiently small. Hence the expression in braces in the last inequality can be made $<-\eta / 2$, for all sufficiently large $n$, by choice of $\varepsilon$. Therefore, from (2.1), we see that 


$$
P\left\{S_{n} \leqq n\left(m_{n}-\eta\right)\right\} \leqq \exp \left\{-\frac{\eta}{2 \sqrt{\varepsilon}}\right\},
$$

for all sufficiently large $n$. Since $\varepsilon$ can be chosen arbitrarily small we are led to the conclusion that

$$
P\left\{S_{n} \leqq n\left(m_{n}-\eta\right)\right\} \rightarrow 0 \quad \text { as } n \rightarrow \infty
$$

for every $\eta>0$.

Let $\rho>0$ be arbitrarily small. Since $E S_{n}=n m_{n}$ we have that

$$
\begin{aligned}
n m_{n} \geqq n\left(m_{n}\right. & +\rho) P\left\{S_{n} \geqq n\left(m_{n}+\rho\right)\right\} \\
& +n\left(m_{n}-\eta\right) P\left\{n\left(m_{n}-\eta\right)<S_{n}<n\left(m_{n}+\rho\right)\right\},
\end{aligned}
$$

and from this inequality it follows that

$$
(\rho+\eta) P\left\{S_{n} \geqq n\left(m_{n}+\rho\right)\right\}<\eta+m_{n} P\left\{S_{n} \leqq n\left(m_{n}-\eta\right)\right\} .
$$

If we choose $\eta$ arbitrarily small, and observe once again that $m_{n}$ is bounded, we can infer from (2.5) and (2.6) that

$$
P\left\{S_{n} \geqq n\left(m_{n}+\rho\right)\right\} \rightarrow 0 \quad \text { as } n \rightarrow \infty
$$

for every $\rho>0$. The coupling of (2.5) and (2.7) produce the desired conclusion that for every $\eta>0$

$$
P\left\{\left|\frac{S_{n}}{n}-m_{n}\right| \geqq \eta\right\} \rightarrow 0 \quad \text { as } n \rightarrow \infty .
$$

We remark that (2.8) proves the lemma for the case when the $\left\{X_{n}\right\}$ are nonnegative (see the Corollary 2 quoted on page 141 of Gnedenko and Kolmogorov (1954)). We turn now to the general case, and begin by defining

$$
\begin{aligned}
X_{n}^{+} & =X_{n}, & & \text { if } X_{n} \geqq 0, \\
& =0, & & \text { otherwise },
\end{aligned}
$$

and $X_{n}^{-}=X_{n}^{+}-X_{n}$. Thus both $X_{n}^{+}$and $X_{n}^{-}$are nonnegative random variables and we shall write $\mu_{n}^{+}=E X_{n}^{+}, \mu_{n}^{-}=E X_{n}^{-}, m_{n}^{+}=n^{-1}\left(\mu_{1}^{+}+\right.$ $\left.\mu_{2}^{+}+\cdots+\mu_{n}^{+}\right), m_{n}^{--}=n^{-1}\left(\mu_{1}^{-}+\mu_{2}^{-}+\cdots+\mu_{n}^{-}\right)$, and, of course, $m_{n}=$ $n^{-1}\left(\mu_{1}+\mu_{2}+\cdots+\mu_{n}\right)$.

Since (T6)(a) holds for $k=-1$, the mean of $K(x)$ is finite; if we call this mean $-\kappa$ then it also follows from (T6)(a) that $\mu_{n}^{-} \leqq \kappa$ for all $n$, and hence $m_{n}^{-} \leqq \kappa$. Moreover, the fact that $K(x) \geqq F_{n}(x)$, for all $n$ and all $x$, ensures that (1.7) will hold when the $\left\{F_{n}(x)\right\}$ in that condition are replaced by the corresponding distribution functions of the variables $\left\{X_{n}^{-}\right\}$. We can now infer from the result established for nonnegative random variables at the start of this proof that, if we write $S_{n}^{-}=X_{1}^{-}+X_{2}^{-}+\cdots+X_{n}^{-}$, 


$$
P\left\{\left|\frac{S_{n}^{-}}{n}-m_{n}^{-}\right| \geqq \eta\right\} \rightarrow 0 \quad \text { as } n \rightarrow \infty,
$$

for every $\eta>0$.

Let us turn now to a consideration of $S_{n}^{+}=X_{1}^{+}+X_{2}^{+}+\cdots+X_{n}^{+}$. We note first that, since $\mu_{n}^{+}-\mu_{n}^{-}=\mu_{n}$, we have $m_{n}^{+}=m_{n}^{-}+m_{n}$. But we have just shown that $m_{n}^{-}$is bounded by $\kappa$; and we may now suppose $m_{n} \rightarrow \mu$ as $n \rightarrow \infty$, by (1.6). Thus $m_{n}^{+}$is a bounded function of the integer $n$, and we can appeal to our preliminary result to deduce that

$$
P\left\{\left|\frac{S_{n}^{+}}{n}-m_{n}^{+}\right| \geqq \eta\right\} \rightarrow 0 \quad n \rightarrow \infty,
$$

for every $\eta>0$. The lemma follows from (2.9) and (2.10).

LEMma 3. Under the conditions of Lemma 2,

$$
\int_{\mu}^{\infty}\left\{1-G_{n}(n x)\right\} d x \rightarrow 0 \quad \text { as } x \rightarrow \infty .
$$

Proof. It is easy to verify that

$$
\begin{aligned}
m_{n}= & \int_{-\infty}^{+\infty}\left\{U(x)-G_{n}(n x)\right\} d x, \\
= & \int_{\mu}^{\infty}\left\{1-G_{n}(n x)\right\} d x+\int_{0}^{\mu}\left\{1-G_{n}(n x)\right\} d x \\
& -\int_{-\kappa}^{0} G_{n}(n x) d x-\int_{-\infty}^{-\kappa} G_{n}(n x) d x, \\
= & A_{n}+B_{n}-C_{n}-D_{n}, \quad \text { say. }
\end{aligned}
$$

By Lemma 2, $G_{n}(n x) \rightarrow 0$ as $n \rightarrow \infty$, for all $x<\mu$. Thus $B_{n} \rightarrow \mu$ and $C_{n} \rightarrow 0$ as $n \rightarrow \infty$, by bounded convergence. But $m_{n} \rightarrow \mu$, by (1.6). Thus we can see from (2.12) that in order to establish the required result, $A_{n} \rightarrow 0$, we need only prove that $D_{n} \rightarrow 0$. In the case when the $\left\{X_{n}\right\}$ are nonnegative there is, of course, no need for further argument.

Write $K_{n}(x)$ for the familiar $n$-fold Stieltjes convolution of $K(x)$ with itself. Then plainly, since $K(x) \geqq F_{n}(x)$ for all $n$ and all $x$, $K_{n}(x) \geqq G_{n}(x)$ for all $n$ and all $x$. Thus it will be enough if we can prove that

$$
\int_{-\infty}^{-\kappa} K_{n}(n x) d x \rightarrow 0 \quad \text { as } n \rightarrow \infty .
$$

However, 


$$
\int_{-\infty}^{0} K_{n}(n x) d x=\kappa, \quad \text { for all } n,
$$

so that (2.13) would follow if we proved that

$$
\int_{-\kappa}^{0} K_{n}(n x) d x \rightarrow \kappa \quad \text { as } n \rightarrow \infty .
$$

To prove (2.14) we need only remark that, by the weak law of large numbers for identically distributed random variables, $K_{n}(n x) \rightarrow 1$ as $n \rightarrow \infty$ for all $x>-\kappa$; thus (2.14) follows from the theorem on bounded convergence. This proves the lemma.

LEMMA 4. If the nonnegative constants $\left\{a_{n}\right\}$ satisfy (1.3) then, as $s \rightarrow 0+$,

$$
\sum_{n=1}^{\infty} a_{n} e^{-\mu s n} \sim \frac{\alpha}{\mu^{\gamma} s^{\gamma}} L\left(\frac{1}{s}\right)
$$

Proof. Plainly, $e^{-\mu s} \rightarrow 1-0$ as $s \rightarrow 0+$. Therefore, as $s \rightarrow 0+$,

$$
\begin{aligned}
\sum_{n=1}^{\infty} a_{n} e^{-\mu s n} & \sim \frac{\alpha}{\left(1-e^{-\mu s}\right)^{\gamma}} L\left(\frac{1}{1-e^{-\mu s}}\right) \\
& \sim \frac{\alpha}{\mu^{\gamma} s^{\gamma}} L\left(\frac{1}{1-e^{-\mu s}}\right) .
\end{aligned}
$$

But, as we have remarked in our introduction, $L(r x) / L(x) \rightarrow 1$ as $x \rightarrow \infty$ uniformly for $r$ in any interval not containing 0 . Thus it transpires that

$$
L\left(\frac{1}{1-e^{-\mu s}}\right) \sim L\left(\frac{1}{s}\right)
$$

as $s \rightarrow 0+$, since $1-e^{-\mu s} \sim \mu s$ for small $s$. Thus the lemma is proved.

Lemma 5. Under the same conditions as Lemma 4, as $s \rightarrow 0+$,

$$
\sum_{n=1}^{\infty} n a_{n} e^{-\mu s n} \sim \frac{\gamma \alpha}{(\mu s)^{\gamma+1}} L\left(\frac{1}{s}\right) .
$$

Proof. Choose $\eta, 0<\eta<1$. Then

$$
\mu n e^{-\mu n s}<\frac{e^{-\mu s n \eta}-e^{-\mu s n}}{(1-\eta) s} .
$$

Thus

$$
\mu \cdot \sum_{n=1}^{\infty} n a_{n} e^{-\mu n s}<\frac{\sum_{n=1}^{\infty} a_{n} e^{-\mu s n \eta}-\sum_{n=1}^{\infty} a_{n} e^{-\mu s n}}{(1-\eta) s}
$$


and so

$$
\frac{\mu s^{\gamma+1}}{L\left(\frac{1}{s}\right)} \sum_{n=1}^{\infty} n a_{n} e^{-\mu s n}<\frac{s^{\gamma}}{(1-\eta) L\left(\frac{1}{s}\right)}\left\{\sum_{n=1}^{\infty} a_{n} e^{-\mu s n \eta}-\sum_{n=1}^{\infty} a_{n} e^{-\mu s n}\right\} .
$$

It follows therefore, from Lemma 4, that

$$
\limsup _{s \rightarrow 0+} \frac{\mu s^{\gamma+1}}{L\left(\frac{1}{s}\right)} \sum_{n=1}^{\infty} n a_{n} e^{-\mu s n} \leqq \frac{\alpha}{\mu^{\gamma}}\left\{\frac{\eta^{-\gamma}-1}{1-\eta}\right\} .
$$

If we let $\eta \rightarrow 1-0$ in (2.15) we obtain

$$
\limsup _{s \rightarrow 0+} \frac{\mu s^{\gamma+1}}{L\left(\frac{1}{s}\right)} \sum_{n=1}^{\infty} n a_{n} e^{-} \mu^{s n} \leqq \frac{\alpha \gamma}{\mu^{\gamma}} .
$$

Similarly, by taking $\eta>1$ and using the fact that

$$
\mu n e^{-\mu n s}>\frac{e^{-\mu s n}-e^{-\mu s n \eta}}{\eta-1}
$$

we can show

$$
\liminf _{s \rightarrow 0+} \frac{\mu s^{\gamma+1}}{L\left(\frac{1}{s}\right)} \sum_{n=1}^{\infty} n a_{n} e^{-\mu s n} \geqq \frac{\alpha \gamma}{\mu^{\gamma}}
$$

The lemma follows from (2.16) and (2.17).

3. Proof of Theorem 1. We shall write $\beta$ for an upper bound to the numbers $\left\{a_{n} / n^{k}\right\}$, where $k$ is the index of the nonnegative coefficient sequence $\left\{a_{n}\right\}$; we shall also write $\eta>0$ for an arbitrary small number; it is supposed that $\eta<\mu$.

Consider, to begin with,

$$
\begin{aligned}
K_{n} & =\int_{n \eta}^{n \mu} e^{-s x} G_{n}(x) d x, \\
& =n \int_{\eta}^{\mu} e^{-n s x} G_{n}(n x) d x .
\end{aligned}
$$

Evidently,

$$
0 \leqq K_{n} \leqq n e^{-n \eta s} \int_{\eta}^{\mu} G_{n}(n x) d x
$$

But $G_{n}(n x) \rightarrow 0$ as $n \rightarrow \infty$, for all $x<\mu$, by Lemma 2. Hence we can appeal to bounded convergence and write 


$$
K_{n}=n e^{-n \eta s} \delta_{n}^{\prime},
$$

where $\delta_{n}^{\prime} \rightarrow 0$ as $n \rightarrow \infty$, uniformly in $s \geqq 0$.

Next consider

$$
\begin{aligned}
L_{n} & =\int_{n \mu}^{\infty} e^{-s x}\left\{1-G_{n}(x)\right\} d x \\
& =n \int_{\mu}^{\infty} e^{-n s x}\left\{1-G_{n}(n x\} d x .\right.
\end{aligned}
$$

In view of Lemma 3 and the assumption that $\eta<\mu$ we may thus conclude that

$$
L_{n}=n e^{-n \eta s} \delta_{n}^{\prime \prime} \text {, }
$$

where $\delta_{n}^{\prime \prime} \rightarrow 0$ as $n \rightarrow \infty$, uniformly in $s \geqq 0$.

Thus, if we write $\delta_{n}=\delta_{n}^{\prime}-\delta_{n}^{\prime \prime}$,

$$
\sum_{n=1}^{\infty} a_{n}\left(L_{n}-K_{n}\right)=\sum_{n=1}^{\infty} n a_{n} \delta_{n} e^{-n \eta s} \text {. }
$$

Given an arbitrary $\varepsilon>0$, we can find $n_{0}(\varepsilon)$ such that $\left|\delta_{n}\right|<\varepsilon$ for all $n>n_{0}$. Moreover we can assume that $s^{-\gamma} L\left(s^{-1}\right) \rightarrow \infty$ as $s \rightarrow 0+$, since we suppose $\sum a_{n}$ to be divergent. Thus

$$
\left|\sum_{n=1}^{\infty} n a_{n} \delta_{n} e^{-n \eta s}\right|<\sum_{n=1}^{n_{0}} n a_{n}\left|\delta_{n}\right| e^{-n \eta s}+\varepsilon \sum_{n=1}^{\infty} n a_{n} e^{-n \eta s} .
$$

Therefore, by Lemma 5 ,

$$
\limsup _{s \rightarrow 0+} \frac{(\mu s)^{\gamma+1}}{L\left(\frac{1}{s}\right)}\left|\sum_{n=1}^{\infty} n a_{n} \delta_{n} e^{-n \eta s}\right| \leqq \varepsilon \gamma \alpha .
$$

But $\varepsilon$ is arbitrary, and we can therefore deduce from (3.7) and (3.5) that, as $s \rightarrow 0+$,

$$
\frac{(\mu s)^{\gamma+1}}{L\left(\frac{1}{s}\right)} \sum_{n=1}^{\infty} a_{n}\left(L_{n}-K_{n}\right) \rightarrow 0 .
$$

Now consider the function

$$
H_{\eta}(x)=\sum_{n=1}^{\infty} a_{n} G_{n}(x) U(x-n \eta) .
$$

Evidently $H_{\eta}(x)$ is non-decreasing, since each term in the summation is non-decreasing. We also note that

$$
\begin{aligned}
H_{\eta}(x)= & \sum_{n=1}^{\infty} a_{n} U(x-n \mu) \\
& -\sum_{n=1}^{\infty} a_{n}\left\{U(x-n \mu)-G_{n}(x)\right\} U(x-n \eta) .
\end{aligned}
$$


Let us denote the Laplace transform of a function $A(x)$, say, thus:-

$$
A^{0}(s)=\int_{0}^{\infty} e^{-s x} A(x) d x .
$$

Then, from (3.10), we have

$$
H_{\eta}^{0}(s)=\frac{1}{s} \sum_{n=1}^{\infty} a_{n} e^{-n \mu s}+\sum_{n=1}^{\infty} a_{n}\left(L_{n}-K_{n}\right) ;
$$

the term-by-term integration being justified by monotone convergence.

From (3.11), (3.8), and Lemma 4, it now appears that

$$
\frac{(\mu s)^{\gamma+1}}{L\left(\frac{1}{s}\right)} H_{\eta}^{0}(s) \rightarrow \alpha \mu, \quad \text { as } s \rightarrow 0+\text {. }
$$

An appeal to Doetsch $(1950$, p. 511) then allows the inference

$$
\frac{\mu^{\gamma} \Gamma(1+\gamma)}{t^{\gamma} L(t)} H_{\eta}(t) \rightarrow \alpha \quad \text { as } t \rightarrow \infty .
$$

But, by (3.9),

$$
\sum_{n=1}^{\infty} a_{n} G_{n}(x)=H_{n}(x)+\Psi_{\eta}(x), \quad \text { say },
$$

where

$$
\Psi_{\eta}(x)=\sum_{n=1}^{\infty} a_{n} G_{n}(x)\{1-U(x-n \eta)\} .
$$

If we were to prove that

$$
\lim _{\eta \rightarrow 0} \limsup _{x \rightarrow \infty} \frac{\Psi_{\eta}(x)}{x^{\gamma} L(x)}=0
$$

then the theorem would follow from (3.14) and (3.13). The proof of (3.16) under fairly weak hypotheses is quite involved, however, and we therefore present it in the following two separate sections.

4. Completion of proof under (T6). If $\left\{X_{n}\right\}$ is the renewal sequence under study let us write $X_{n}^{-}=-X_{n}$ when $X_{n}<0, X_{n}^{-}=0$ when $X_{n} \geqq 0$. When (T6) holds we can introduce the distribution function $K(x)$ which, as has already been explained in $\S 1$, may be assumed to have a finite first moment $-\kappa$. Therefore, if we write $\nu_{n}=E X_{n}^{-}$, we have $0 \leqq \nu_{n} \leqq \kappa$ for all $n$.

Let us also write $X_{n}^{+}=X_{n}+X_{n}^{-}, S_{n}^{+}=X_{1}^{+}+X_{2}^{+}+\cdots+X_{n}^{+}$, and $S_{n}^{-}=X_{1}^{-}+X_{2}^{-}+\cdots+X_{n}^{-}$. 
LEMma 6. When (T6) holds we can find $\eta>0, \delta>0$, such that

$$
P\left\{S_{n}^{+} \leqq \nu_{1}+\nu_{2}+\cdots+\nu_{n}+n \eta\right\}=O\left(\frac{1}{n^{k+\delta+1}}\right),
$$

where $k$ is the index of the coefficient sequence $\left\{a_{n}\right\}$.

Proof. Let us write $\bar{\nu}_{n}=n^{-1}\left(\nu_{1}+\nu_{2}+\cdots+\nu_{n}\right)$. Then, for any $t \geqq 0, \eta>0$, it is plain that

$$
\begin{aligned}
P\left\{S_{n}^{+} \leqq n\left(\bar{\nu}_{n}+\eta\right)\right\} & \leqq e^{n\left(\eta+\bar{\nu}_{n}\right) t} E e^{-t S_{n}^{+}} \\
& =e^{n\left(\eta+\bar{\nu}_{n}\right) t} \prod_{j=1}^{n} \Phi_{j}^{+}(t),
\end{aligned}
$$

where

$$
\Phi_{j}^{+}(t)=F_{j}(0+)+\int_{0+}^{\infty} e^{-t x} d F_{j}(x) .
$$

If we now use the familiar inequality already employed in $\$ 2$ we can deduce from (4.2) that

$$
P\left\{S_{n}^{+} \leqq n\left(\bar{\nu}_{n}+\eta\right)\right\} \leqq e^{W_{n}(t)},
$$

where, after some integrations by parts, we now have

$$
W_{n}(t)=n\left(\eta+\bar{\nu}_{n}\right) t-t \sum_{j=1}^{n} \int_{0}^{\infty} e^{-t x}\left\{1-F_{j}(x)\right\} d x .
$$

In this section we are assuming (1.10) to hold, and so, given any fixed $\varepsilon>0$, we have for all sufficiently large $n$ that

$$
\sum_{j=1}^{n} \int_{-\infty}^{\varepsilon n / \log n}\left\{U(x)-F_{j}(x)\right\} d x>2 n \sqrt{(k+1) v \varepsilon},
$$

where $v>\kappa$ is independent of $\varepsilon$.

We can rewrite this last inequality as follows.

$$
\sum_{j=1}^{n} \int_{0}^{\varepsilon n / \log n}\left\{1-F_{j}(x)\right\} d x>n \bar{\nu}_{n}+2 n \sqrt{(k+1) v \varepsilon}
$$

Thus, from (4.4), it is plain that

$$
W_{n}(t) \leqq n\left(\eta+\bar{\nu}_{n}\right) t-n t e^{-t \varepsilon n / \log n}\left(\bar{\nu}_{n}+2 \sqrt{(k+1) v \varepsilon)} .\right.
$$

If, in the latter inequality, we make the substitutions

$$
\begin{gathered}
t=t_{n}=\frac{\lambda \log n}{\varepsilon n}, \\
\lambda=\sqrt{\frac{\varepsilon(k+1)}{\kappa}},
\end{gathered}
$$

and if we note incidentally that $\kappa \geqq \bar{\nu}_{n}$ for all $n$, and $1-e^{-x}<x$ for all $x>0$, then we find that

$$
\frac{W_{n}\left(t_{n}\right)}{\log n}<\frac{\lambda \eta}{\varepsilon}+(k+1)-2 e^{-\lambda}(k+1) \sqrt{\frac{\bar{v}}{\kappa}} .
$$


By taking $\varepsilon$ sufficiently small we can make $e^{-\lambda}$ arbitrarily near unity and thus make

$$
(k+1)-2 e^{-\lambda}(k+1) \sqrt{\frac{v}{\kappa}}<-(k+2 \delta+1)
$$

for some small $\delta>0$ (recall that $v>\kappa$ ). Next choose $\eta$ so small that $\lambda \eta<\varepsilon \delta$ and it follows from (4.6) that

$$
W_{n}\left(t_{n}\right)<-(k+\delta+1) \log n \text {. }
$$

Lemma 6 follows from (4.7) and (4.3).

In what follows we denote the familiar Stieltjes convolution of two distribution functions, say $A(x)$ and $B(x)$, by $A * B(x)$. We denote $A * A(x)$ by $A^{* 2}(x)$, and, generally, $A * A^{* n}(x)$ by $A^{*(n+1)}(x)$, for $n=$ $1,2,3, \cdots$.

Lemma 7. When (T6) holds

$$
\sum_{n=1}^{\infty} n^{k} P\left\{S_{n}^{-}>n\left(\bar{\nu}_{n}+\delta\right)\right\}
$$

is convergent for every $\delta>0$.

Proof. Define $Z_{n}=\delta / 2+\nu_{n}-X_{n}^{-}$and write $L_{n}(x)=P\left\{Z_{n} \leqq x\right\}$. If we recall that $\nu_{n} \leqq \kappa$ for all $n$, then we easily see that

$$
U\left(x-\frac{1}{2} \delta-\kappa\right) \leqq L_{n}(x) \leqq K(x) .
$$

This proves that $Z_{n}$ is a stochastically stable sequence as defined by Smith (1962), whose Theorem 7 allows us to draw the following conclusion. that

For every integer $p$ there is a distribution function $K_{p}(x)$ such

$$
P\left\{Z_{n}+Z_{n+1}+\cdots+Z_{n+p-1} \leqq p x\right\} \leqq K_{p}(x)
$$

for all $n$ and all $x$, where

$$
I_{p} \equiv \int_{-\infty}^{0} K_{p}(x) d x
$$

is finite for all $p$, and $I_{p} \rightarrow 0$ as $p \rightarrow \infty$.

Thus we can find $p_{0}(\delta / 2)$ such that $I_{p_{0}}<\delta / 2$. If $Y$ is a random variable with distribution function $K_{p_{0}}$ then it follows from (4.9) that $E Y>-\delta / 2$. Moreover, it is clear that $E\left\{|\min (0, Y)|^{k+2}\right\}<\infty$, since we can certainly suppose $K_{p}(x) \leqq K^{* p}(p x)$.

Write $M(x)$ for the supremum of $P\left\{Z_{1}+Z_{2}+\cdots+Z_{r} \leqq p_{0} x\right\}$ for 
$r=1,2, \cdots, p_{0}-1$. Then if $Y_{0}$ is a random variable with distribution function $M(x)$ it is also apparent that $E\left\{\left|\min \left(0, Y_{0}\right)\right|^{k+2}\right\}<\infty$.

Now choose and fix $r=0$, or 1 , or $2, \cdots$, or $p_{0}-1$. It follows from what we have established so far that

$$
P\left\{\sum_{j=1}^{n p_{0}+r} Z_{j} \leqq p_{0} x\right\} \leqq M * K_{p_{0}}^{* n}(x)
$$

Let $Y_{1}, Y_{2}, \cdots$ be a sequence of independent random variables, identically distributed, with distribution function $K_{p_{0}}(x)$; let $Y_{1}, Y_{2}, \cdots$ be independent of $Y_{0}$. Then $E\left(Y_{j}+\delta / 2\right)>0$, for $j=1,2,3, \cdots$ and $E\left\{\left|\min \left(0, Y_{j}+1 / 2 \delta\right)\right|^{k+2}\right\}<\infty$ for $j=0,1,2, \cdots$. Thus it follows from Theorem $\mathrm{A}$, quoted in $\S 1$, that

$$
\sum_{n=1}^{\infty} n^{k} P\left\{Y_{0}+\sum_{j=1}^{n}\left(Y_{j}+\delta / 2\right) \leqq 0\right\}<\infty,
$$

that is,

$$
\sum_{j=1}^{\infty} n^{k} M * K_{p_{0}}^{* n}\left(-\frac{1}{2} n \delta\right)<\infty
$$

From (4.10) and (4.11) we conclude that

$$
\sum_{n=1}^{\infty} n^{k} P\left\{\left(n p_{0}+r\right)\left(\frac{1}{2} \delta+\bar{\nu}_{n p_{0}+r}\right) \leqq S_{n p_{0}+r}^{-}-\frac{1}{2} n p_{0} \delta\right\}<\infty,
$$

whence,

$$
\sum_{n=1}^{\infty} n^{k} P\left\{S_{n p_{0}+r}^{-} \geqq\left(n p_{0}+r\right)\left(\delta+\bar{\nu}_{n p_{0}+r}\right)\right\}<\infty .
$$

The lemma follows from (4.12) by letting $r=0,1,2, \cdots, p_{0}-1$ in turn.

LeMMA 8. When (T6) holds we can find $\eta>0$ such that

$$
\sum_{n=1}^{\infty} n^{k} G_{n}(n \eta)<\infty
$$

Proof. We observe that

$$
\begin{aligned}
P\left\{S_{n} \leqq n \eta\right\}= & P\left\{S_{n}^{+} \leqq n \eta+S_{n}^{-}\right\} \\
\leqq & P\left\{S_{n}^{+} \leqq n \eta+S_{n}^{-}, S_{n}^{-}<n\left(\delta+\bar{\nu}_{n}\right)\right\} \\
& +P\left\{S_{n}^{-} \geqq n\left(\delta+\bar{\nu}_{n}\right)\right\}
\end{aligned}
$$

for every $\delta>0$. Hence

$$
P\left\{S_{n} \leqq n \eta\right\} \leqq P\left\{S_{n}^{+} \leqq n\left(\eta+\delta+\bar{\nu}_{n}\right)\right\}+P\left\{S_{n}^{-} \geqq n\left(\delta+\bar{\nu}_{n}\right)\right\} .
$$


The lemma now follows from Lemmas 6 and 7 if we make $\eta+\delta$ sufficiently small.

The proof of (3.16) is now straightforward. We see from (3.15) that

$$
\Psi_{\eta}(x) \leqq \sum_{n=1}^{\infty} a_{n} G_{n}(n \eta)
$$

for all $x$. Therefore, since $k$ is the index of the $\left\{a_{n}\right\}$ sequence, it follows from Lemma 8 that $\Psi_{\eta}(x)$ is bounded above. Since $x^{\gamma} L(x) \rightarrow \infty$ as $x \rightarrow \infty$, the truth of (3.16) is established.

5. Completion of proof under (T5). We begin by showing that, once we have proved (1.9), we can assume, with no loss of generality, certain convenient properties for the function $l(n)$. All that actually matters are the values taken by $l(n)$ for integer values of $n$; but we may clearly assume $l(x)$ to be a continuous function defined for all $x \geqq 1$. More to the point, we observe that if (1.9) holds for the function $l(x)$ then it also holds for any function $l_{1}(x) \leqq l(x)$. In this connection we prove the following;

Lemma 9. If, for $x \geqq 1, l(x)$ is an unbounded, continuous, and non-decreasing function of $x$ then we can find another such function $l_{1}(x) \leqq l(x)$, defined on the same domain, with the additional property that $l_{1}(x) / x$ is non-increasing for all sufficiently large $x$, and tends to zero as $x \rightarrow \infty$.

Proof. For $x \geqq 1$ define

$$
l_{1}(x)=\log x+\inf _{1 \leqq y \leqq x}\{l(y)-\log (y)\} .
$$

We shall show that $l_{1}(x)$ has all the requisite properties.

To begin with, since $l(y)-\log y$ is a continuous function in [1, $x]$ it attains its lower bound; we shall write $y(x) \leqq x$ for the largest $y$-value at which this lower bound is attained. Then

$$
l_{1}(x)=\log x+l(y(x))-\log y(x) .
$$

Evidently $y(x)$ is a non-decreasing function of $x$. If $y(x) \rightarrow \infty$ as $x \rightarrow \infty$ then the fact that $l(x)$ is unbounded shows, in (5.2), that $l_{1}(x)$ is also unbounded; if $y(x)$ tends to a finite limit as $x \rightarrow \infty$, then the fact that $\log x$ is unbounded shows, also in (5.2), that $l_{1}(x)$ is unbounded. Incidentally, it is an easy deduction from (5.1) that $l_{1}(x) \leqq l(x)$.

Next choose an arbitrary value of $x, x_{1}$ say. Our argument will be given in two cases. 
Case $y\left(x_{1}\right)<x_{1}$. The continuity of $l(y)-\log y$ ensures the existence of some open interval $G$, containing $x_{1}$, within which $y(x)=$ $y\left(x_{1}\right)$. Hence, in $G$,

$$
l_{1}(x)=\log x-\log x_{1}+l_{1}\left(x_{1}\right) ;
$$

from this equation it is clear that $l_{1}(x)$ is increasing in $G$ and, by simple differentiation, $l_{1}(x) / x$ is decreasing in $G$.

Case $y\left(x_{1}\right)=x_{1}$. In this case, for any $h>0, y\left(x_{1}+h\right) \geqq x_{1}$; thus $l\left(y\left(x_{1}+h\right)\right) \geqq l\left(x_{1}\right)=l_{1}\left(x_{1}\right)$. Hence, by (5.2),

$$
l_{1}\left(x_{1}+h\right) \geqq \log \left(x_{1}+h\right)+l_{1}\left(x_{1}\right)-\log \left(y\left(x_{1}+h\right)\right) .
$$

Since $x_{1}+h \geqq y\left(x_{1}+h\right)$, it follows from (5.3) that $l_{1}\left(x_{1}+h\right) \geqq l_{1}\left(x_{1}\right)$, i.e. $l_{1}(x)$ is increasing at $x_{1}$. But, from (5.1),

$$
l_{1}\left(x_{1}+h\right)-\log \left(x_{1}+h\right) \leqq l_{1}\left(x_{1}\right)-\log x_{1}
$$

from which we can infer that

$$
\frac{l_{1}\left(x_{1}+h\right)}{x_{1}+h}-\frac{l_{1}\left(x_{1}\right)}{x_{1}} \leqq-\frac{h l_{1}\left(x_{1}\right)}{x_{1}\left(x_{1}+h\right)}+\frac{h}{x_{1}\left(x_{1}+h\right)} .
$$

The right hand side of (5.4) is negative for all sufficiently large $x_{1}$, because $l_{1}(x)$ is unbounded and non-decreasing. Thus $l_{1}(x) / x$ is nondecreasing at $x_{1}$.

Finally, we remark that

$$
l_{1}(x) \leqq \log x+l(1),
$$

from which it is obvious that $l_{1}(x) / x \rightarrow 0$ as $x \rightarrow \infty$.

Let us next establish that conditions (T1) and (T2) of Theorem 1 do indeed imply the existence of some unbounded increasing function $l(n)$ for which (1.9) holds. We see that since (1.7) is true for every $\varepsilon>0$, there must be an unbounded increasing function $w(n)$, say, such that

$$
\frac{1}{n} \sum_{r=1}^{\infty} \int_{n / w(n)}^{\infty}\left\{1-F_{r}(x)\right\} d x \rightarrow 0 \quad \text { as } n \rightarrow \infty .
$$

Therefore, because of (1.6), it follows that

$$
\frac{1}{n} \sum_{r=1}^{n} \int_{0}^{n / w(n)}\left\{1-F_{r}(x)\right\} d x \rightarrow \mu \quad \text { as } n \rightarrow \infty .
$$

Let $l(n)$ be some other unbounded increasing function; we leave this function somewhat arbitrary for the moment except for the supposition that it increases very much more slowly than $w(n)$. Let $t(n)$ be such that $r / l(r) \geqq n / w(n)$ for all $r>t(n)$. Then we can infer 
that, for any positive $\varepsilon$, and all sufficiently large $n$,

$$
\begin{aligned}
(\mu-\varepsilon) & <\frac{1}{n} \sum_{r=1}^{t(n)} \int_{0}^{n / w(n)}\left\{1-F_{r}(x)\right\} d x+\frac{1}{n} \sum_{r=t(n)+1}^{n} \int_{0}^{r / l(r)}\left\{1-F_{r}(x)\right\} d x \\
& =T_{1}(n)+T_{2}(n), \quad \text { say . }
\end{aligned}
$$

But

$$
\frac{1}{n} \sum_{r=1}^{t(n)} \int_{0}^{n / w(n)}\left\{1-F_{r}(x)\right\} d x \leqq \frac{1}{n}\left(\mu_{1}+\mu_{2}+\cdots+\mu_{t(n)}\right),
$$

and so we could conclude from (1.6) that $T_{1}(n) \rightarrow 0$ as $n \rightarrow \infty$ if only we could be sure that $t(n) / n \rightarrow 0$. It would than follow that $T_{2}(n)>(\mu-2 \varepsilon)$ for all large $n$; the desired conclusion (1.9) would then be proved, in view of the arbitrariness of $\varepsilon$.

For large $n$, since $w(n)$ is increasing,

$$
w(n) \geqq w\left(n \frac{\log w(n)}{w(n)}\right)
$$

and so, if we put $s(n)=n(\log w(n)) / w(n)$,

$$
\frac{s(n)}{\log w(s(n))} \geqq \frac{n}{w(n)} .
$$

It is clear from Lemma 9 that we may assume $n / w(n)$ to be increasing for all large $n$; from this it is easily seen that $n /(\log w(n))$ is also increasing for all large $n$. Therefore, if we let $l(n)=\log \mathrm{w}(n)$ we have that $r / l(r) \geqq n / w(n)$ for all $r \geqq s(n)$. Hence $t(n) \leqq s(n)$, and it is plain that $s(n) / n \rightarrow 0$ as $n \rightarrow \infty$; thus we have a function $l(n)$ which exhibits the desired behavior, and (1.9) is proved.

We now turn to the proof of (3.16) under the condition (T5) that the $\left\{X_{n}\right\}$ are nonnegative; for this proof we may, by the immediately preceding discussion, assume that $x / l(x)$ is unbounded and non-decreasing. We can then define $r^{*}=r^{*}(x)$ as the greatest integer such that $r^{*} / l\left(r^{*}\right) \leqq x$. We also write $s^{*}=s^{*}(x)=r^{*}((1+e) x)$; thus $s^{*}(x)$ is the greatest positive integer such that $s^{*} / l\left(s^{*}\right) \leqq(1+e) x$.

Choose a large positive $C$ and consider the following three cases, in all of which $x$ is assumed to be large.

(i) $c x \leqq n \leqq r^{*}(x)$. By considerations similar to those in the proof of Lemma 2 we have

$$
G_{n}(x) \leqq e^{W_{n}(t)},
$$

where

$$
W_{n}(t)=t x-t \sum_{j=1}^{n} \int_{0}^{\infty} e^{-t u}\left\{1-F_{j}(u)\right\} d u \text {. }
$$


If we substitute $t=1 /(2 x)$ and truncate the integrals at $x$ in (5.6) then we find

$$
W_{n}(1 /(2 x)) \leqq \frac{1}{2}-\frac{e^{-1 / 2}}{2 x} \sum_{j=1}^{n} \int_{0}^{x}\left\{1-F_{j}(u)\right\} d u .
$$

Since (T5) is assumed to hold we can, by (1.9), find $\delta>0$ such that

$$
\sum_{j=1}^{n} \int_{0}^{j / l(j)}\left\{1-F_{j}(u)\right\} d u>n \delta
$$

for all sufficiently large $n$. But $n \leqq r^{*}$ so that (since $x / l(x)$ is nondecreasing) $j / l(j) \leqq x$ for $j=1,2, \cdots, n$. (Actually we have only shown that $x / l(x)$ is non-decreasing for all sufficiently large $x$; but this is adequate for our purpose if we note that $x / l(x) \rightarrow \infty$ as $x \rightarrow \infty$ and assume $n$ large). Hence we can infer from (5.8) that

$$
\sum_{j=1}^{n} \int_{0}^{x}\left\{1-F_{j}(u)\right\} d u>n \delta
$$

and so, from (5.7), that

$$
W_{n}(1 /(2 x)) \leqq \frac{1}{2}-\frac{e^{-1 / 2} n \delta}{2 x} .
$$

If we use this last inequality in (5.5) we deduce that

$$
G_{n}(x) \leqq \exp \left\{\frac{1}{2}-\frac{e^{-1 / 2} n \delta}{2 x}\right\}, \quad n \leqq r^{*} .
$$

(ii) $r^{*}(x)<n \leqq s^{*}(x)$. For this case we modify the kind of inequality we have been using on $G_{n}(x)$. Plainly

$$
\begin{aligned}
P\left\{S_{n} \leqq x\right\} \leqq P\left\{X_{j}\right. & \leqq x \text { for all } j\}=\prod_{j=1}^{n} F_{j}(x) \\
& \leqq \exp \left\{-\sum_{j=1}^{n}\left[1-F_{j}(x)\right]\right\}
\end{aligned}
$$

By forming the geometric mean of this last inequality and (5.5) we obtain a new inequality:

$$
G_{n}(x) \leqq e^{R_{n}(t)},
$$

where

$$
\begin{aligned}
R_{n}(t)= & \frac{1}{2} t x-\frac{1}{2} t \sum_{j=1}^{n} \int_{0}^{\infty} e^{-t u}\left\{1-F_{j}(u)\right\} d u \\
& -\frac{1}{2} \sum_{j=1}^{n}\left\{1-F_{j}(x)\right\} .
\end{aligned}
$$


If we truncate the integrals in (5.12) at $x$ and substitute $t=1 / x$ then we can infer

$$
\begin{aligned}
R_{n}(1 / x) \leqq \frac{1}{2} & -\frac{e^{-1}}{2 x} \sum_{j=1}^{n} \int_{0}^{x}\left\{1-F_{j}(u)\right\} d u \\
& -\frac{1}{2} \sum_{j=r^{*}+1}^{n}\left\{1-F_{j}(x)\right\} .
\end{aligned}
$$

At this point it is convenient to write

$$
\lambda_{j}=\int_{0}^{j / l(j)}\left\{1-F_{j}(u)\right\} d u ;
$$

then (5.9) can be rewritten

$$
\left(\lambda_{1}+\lambda_{2}+\cdots+\lambda_{n}\right)>n \delta, \quad n \text { sufficiently large. }
$$

We shall also write

$$
\alpha_{j}(x)=\int_{0}^{x}\left\{1-F_{j}(u)\right\} d u .
$$

Therefore, for $j>r^{*}$ (and, consequently, $j / l(j)>x$ ) we have

$$
\int_{x}^{j / l(j)}\left\{1-F_{j}(u)\right\} d u=\lambda_{j}-\alpha_{j}(x) .
$$

A consequence of the last equation is that

$$
\left(\frac{j}{l(j)}-x\right)\left(1-F_{j}(x)\right) \geqq \lambda_{j}-\alpha_{j}(x) .
$$

However, if $j \leqq s^{*}(x)$, then $j / l(j) \leqq(1+e) x$, and from this inequality it follows that

$$
\left(\frac{j}{l(j)}-x\right) \leqq e x
$$

Thus we have, from (5.15), that

$$
1-F_{j}(x) \geqq \frac{\lambda_{j}-\alpha_{j}(x)}{e x}, r^{*}<j \leqq s^{*} .
$$

Using the last inequality we can infer from (5.13) that

$$
\begin{aligned}
R_{n}(1 / x)< & \frac{1}{2}-\frac{1}{2 e x} \sum_{1}^{r^{*}} \lambda_{j}-\frac{1}{2 e x} \sum_{r^{*}+1}^{n} \alpha_{j}(x) \\
& -\frac{1}{2 e x} \sum_{r^{*}+1}^{n}\left(\lambda_{j}-\alpha_{j}(x)\right) \\
= & \frac{1}{2}-\frac{1}{2 e x} \sum_{1}^{n} \lambda_{j} \\
< & \frac{1}{2}-\frac{n \delta}{2 e x}
\end{aligned}
$$

by (5.8). 
Therefore, from (5.11), we discover that

$$
G_{n}(x)<\exp \left\{\frac{1}{2}-\frac{n \delta}{2 e x}\right\}, \quad r^{*}<n \leqq s^{*} .
$$

From (5.10) and (5.16) we can conclude that

$$
\sum_{n=C x}^{s^{*}(x)} a_{n} G_{n}(x)<e^{1 / 2} \sum_{n=0 x}^{\infty} a_{n} \exp \left\{-\frac{n \delta}{2 e x}\right\} .
$$

We quote here a theorem which can be immediately deduced from some results of Karamata (Hardy (1949), pp. 166-169, especially Theorems 110, 111).

THEOREM B. Suppose that $\alpha(t)$ is a non-decreasing function of $t$ and that $I(y)=\int_{0}^{\infty} e^{-y t} d \alpha(t)$ is convergent for $y>0$, and that

$$
I(y) \sim \frac{L\left(y^{-1}\right)}{y^{\gamma}}, \quad \text { as } \quad y \rightarrow 0+,
$$

where $\gamma \geqq 0$ and $L(x)$ is a function of slow growth. Then, if $g(x)$ is a continuous function of bounded variation in $(0,1)$, as $y \rightarrow 0+$ we have

(a) in case $\gamma>0$;

$$
\frac{y^{\gamma}}{L\left(y^{-1}\right)} \int_{0}^{\infty} e^{-y t} g\left(e^{-y t}\right) d \alpha(t) \sim \frac{1}{\Gamma(\gamma)} \int_{0}^{\infty} e^{-t} t^{\gamma-1} g\left(e^{-t}\right) d t,
$$

(b) in case $\gamma=0$ :

$$
\frac{1}{L\left(y^{-1}\right)} \int_{0}^{\infty} e^{-y t} g\left(e^{-y t}\right) d \alpha(t) \sim g(1) .
$$

Let us put, in this theorem,

$$
\alpha(t)=\sum_{n \leqq t} \alpha_{n}
$$

Then Lemma 4 shows that a relation like (5.18) holds.

Define

$$
\begin{aligned}
g(x) & =1, & & 0 \leqq x \leqq a, \\
& =2-\frac{x}{a}, & & a \leqq x \leqq 2 a, \\
& =0, & & 2 a \leqq x \leqq 1 .
\end{aligned}
$$

Clearly this $g(x)$ satisfies the conditions of Theorem B. Thus, when $\gamma>0$ we can deduce that 


$$
\begin{aligned}
\limsup _{y \rightarrow 0+} \frac{y^{\gamma}}{L\left(y^{-1}\right)} & \sum_{n \geqq(1 / y) \log (1 / a)} a_{n} e^{-n y} \\
& \leqq \frac{\alpha}{\mu^{\gamma} \Gamma(\gamma)} \int_{\log (1 / 2 a)}^{\infty} e^{-t} t^{\gamma-1} d t .
\end{aligned}
$$

In this last result, substitute $y=\delta /(2 e x)$ and $\log (1 / a)=C \delta /(2 e)$. On being given any prescribed $\varepsilon>0$ we can choose $C$ sufficiently large for us to deduce, via (5.16), that

$$
\limsup _{x \rightarrow \infty} \frac{1}{x^{\gamma} L(x)} \sum_{n=0 x}^{s^{*}(x)} a_{n} G_{n}(x)<\varepsilon .
$$

When $\gamma=0$ a similar result to (5.19) can also be proved by appeal to Theorem B.

(iii) $s^{*}(x)<n$. For this range of values for $n$ we always have $n / l(n)>x$. If we define $R_{n}$ and $\lambda_{n}$ as for case (ii) then by arguments similar to the ones employed in that case we find

$$
\begin{aligned}
R_{n}\left(x^{-1}\right)<\frac{1}{2} & -\frac{1}{2 e x} \sum_{1}^{s^{*}} \lambda_{j}-\frac{1}{2 e x} \sum_{s^{*}+1}^{n} \alpha_{j}(x) \\
& -\frac{1}{2} \sum_{s^{*}+1}^{n}\left\{\frac{\lambda_{j}-\alpha_{j}(x)}{\frac{j}{l(j)}-x}\right\} .
\end{aligned}
$$

However, when $j>s^{*}, j / l(j)>(1+e) x$ and one can infer that

$$
\left(\frac{j}{l(j)}-x\right)>e x
$$

and deduce therefore that

$$
R_{n}\left(x^{-1}\right)<\frac{1}{2}-\frac{1}{2 e x} \sum_{1}^{s^{*}} \lambda_{j}-\frac{1}{2} \sum_{s^{*}+1}^{n}\left\{\frac{\lambda_{j}}{\frac{j}{l(j)}-x}\right\} .
$$

Write

$$
T_{n}=\sum_{s^{*}+1}^{n}\left\{\frac{\lambda_{j}}{\frac{j}{l(j)}-x}\right\}
$$

and

$$
\Lambda_{j}=\lambda_{1}+\lambda_{2}+\cdots+\lambda_{j}
$$


Then

$$
\begin{aligned}
T_{n}= & \frac{\Lambda_{n}}{\frac{n}{l(n)}-x}-\frac{\Lambda_{s^{*}}}{\frac{s^{*}+1}{l\left(s^{*}+1\right)}-x} . \\
& +\sum_{s^{*}+1}^{n-1} \Lambda_{j}\left\{\frac{1}{\frac{j}{l(j)}-x}-\frac{1}{\frac{j+1}{l(j+1)}-x}\right\} .
\end{aligned}
$$

We may assume $s^{*}$ to be large, so that, by $(5.14), \Lambda_{j}>j \delta$ for all $j$ in the range of consideration. Thus

$$
\begin{aligned}
T_{n}> & \frac{n \delta}{\frac{n}{l(n)}-x}-\frac{A_{s^{*}}}{\frac{s^{*}+1}{l\left(s^{*}+1\right)}-x} \\
& +\delta \sum_{s^{*}+1}^{n-1} j\left\{\frac{1}{\frac{j}{l(j)}-x}-\frac{1}{\frac{j+1}{l(j+1)}-x}\right\} \\
= & \left.\frac{\left(\delta s^{*}-\Lambda_{s^{*}}\right)}{\frac{s^{*}+1}{l\left(s^{*}+1\right)}-x}\right\} \sum_{s^{*}+1}^{n}\left\{\frac{1}{\frac{j}{l(j)}-x}\right\} .
\end{aligned}
$$

If we use this last inequality in (5.21), and also make use of (5.20), then we find

$$
\begin{aligned}
2 R_{n}\left(x^{-1}\right) & <1-\frac{s^{*} \delta}{\frac{s^{*}+1}{l\left(s^{*}+1\right)}-x}-\delta \sum_{s^{*}+1}^{n}\left\{\frac{1}{\left.\frac{j}{l(j)}-x\right\}}\right\} \\
& <1-\frac{s^{*} \delta}{\frac{s^{*}+1}{l\left(s^{*}+1\right)}-x}-\delta \sum_{s^{*}+1}^{n} \frac{l(j)}{j} \\
& <1-\frac{s^{*} \delta}{\frac{s^{*}+1}{l\left(s^{*}+1\right)}-x}-\delta l\left(s^{*}+1\right) \log \left(\frac{n+1}{s^{*}+1}\right) .
\end{aligned}
$$

We may therefore conclude, from (5.11), that

$$
G_{n}(x)<\left(\frac{s^{*}+1}{n+1}\right)^{(1 / 2) \delta l\left(s^{*}+1\right)} e^{\psi(x)}, n>s^{*},
$$

where

$$
\psi(x)=\frac{1}{2}-\frac{s^{*} \delta}{2\left(\frac{s^{*}+1}{l\left(s^{*}+1\right)}-x\right)} .
$$

At this point in our argument we need information about the order of magnitude of 


$$
U_{N}=\sum_{N}^{\infty} \frac{a_{n}}{n^{\rho}}
$$

for $\rho>\gamma+2$. To this end, define $\alpha(t)=\Sigma_{n \leqq t} a_{n}$, as before. Then there is some constant $c$ such that $\alpha(t)<c t^{\gamma} L(t)$ for all large $t$, by (1.4).

Evidently

$$
\begin{aligned}
U_{N} & =\int_{N-0}^{\infty} \frac{1}{\mu^{\rho}} d \alpha(u) . \\
& =\left[\frac{1}{u^{\rho}} \alpha(u)\right]_{N-0}^{\infty}+\rho \int_{N}^{\infty} \frac{\alpha(u)}{u^{\rho+1}} d u \\
& <c \rho \int_{N}^{\infty} \frac{L(u)}{u^{\rho-\gamma+1}} d u,
\end{aligned}
$$

if we ignore a negative form. Hence, by an obvious substitution in the integral, we have

$$
U_{N}<\frac{c \rho L(N)}{N^{\rho-\gamma}} \int_{1}^{\infty} \frac{L(N v)}{L(N)} \frac{d v}{v^{\rho-\gamma+1}} .
$$

But, from (1.2),

$$
\frac{L(N v)}{L(N)}=\frac{a(N v)}{a(N)} \cdot \frac{1}{v} \cdot \exp \left\{\int_{N}^{N v} \frac{a(u)}{u} d u\right\},
$$

where $a(x) \rightarrow 1$, as $x \rightarrow \infty$. Therefore, given an arbitrarily small $\varepsilon>0$, we can choose $N$ so large that for all $v \geqq 1$

$$
\begin{aligned}
\frac{L(N v)}{L(N)} & <\frac{(1+\varepsilon)}{v} \exp \left\{(1+\varepsilon) \int_{N}^{N v} \frac{d u}{u}\right\} \\
& =(1+\varepsilon) v^{\varepsilon} .
\end{aligned}
$$

Hence we can appeal to dominated convergence to infer that

$$
\int_{1}^{\infty} \frac{L(N v)}{L(N)} \frac{d v}{v^{\rho-\gamma+1}} \rightarrow \int_{1}^{\infty} \frac{d v}{v^{\rho-\gamma+1}}
$$

as $N \rightarrow \infty$. It then transpires, from (5.23), that

$$
U_{N}=O\left(\frac{L(N)}{N^{\rho-\gamma}}\right)
$$

For all sufficiently large $x$ we shall have $(1 / 2) \delta l\left(s^{*}+1\right)>\gamma+2$, and hence may infer from (5.22) and (5.24) that

$$
\sum_{n=s^{*}+1}^{\infty} a_{n} G_{n}(x)=O\left(\left(s^{*}+1\right)^{\gamma} L\left(s^{*}+1\right) e^{\psi(x)}\right)
$$

and hence that 


$$
\frac{1}{x^{\gamma} L(x)} \sum_{n=s^{*}+1}^{\infty} a_{n} G_{n}(x)=O\left(\left(\frac{s^{*}+1}{x}\right)^{\gamma} \frac{L\left(s^{*}+1\right)}{L(x)} e^{\gamma^{\gamma}(x)}\right) .
$$

Since

$$
\begin{aligned}
\frac{s^{*}+1}{l\left(s^{*}+1\right)} & =\left(\frac{s^{*}+1}{s^{*}}\right)\left(\frac{l\left(s^{*}\right)}{l\left(s^{*}+1\right)}\right)\left(\frac{s^{*}}{l\left(s^{*}\right)}\right) \\
& \leqq\left(\frac{s^{*}+1}{s^{*}}\right)(1+e) x,
\end{aligned}
$$

it is clear that for all large $x$

$$
\frac{s^{*}+1}{l\left(s^{*}+1\right)}<(1+2 e) x .
$$

Therefore, from the definition of $\psi(x)$ which follows (5.22) we infer that

$$
\psi(x)<\frac{1}{2}-\frac{s^{*} \delta}{4 e x} .
$$

In addition, we can deduce from (1.2) that

$$
\frac{L\left(s^{*}+1\right)}{L(x)}=\frac{a\left(s^{*}+1\right)}{a(x)}\left(\frac{x}{s^{*}+1}\right) \exp \left\{\int_{x}^{s^{*}+1} \frac{a(u)}{u} d u\right\}
$$

and so,

$$
\frac{L\left(s^{*}+1\right)}{L(x)}=O\left(\left(\frac{s^{*}+1}{x}\right)^{\varepsilon}\right)
$$

for any $\varepsilon>0$.

If we combine (5.26) and (5.27) with (5.25) we discover that

$$
\frac{1}{x^{\gamma} L(x)} \sum_{n=s^{*}+1}^{\infty} a_{n} G_{n}(x)=O\left(\left(\frac{s^{*}+1}{x}\right)^{\gamma+\varepsilon} \exp \left(-\frac{s^{*} \delta}{4 e x}\right)\right) .
$$

But $\left(s^{*}+1\right)>(1+e) x l\left(s^{*}+1\right)$, so that $\left(s^{*}+1\right) / x \rightarrow \infty$ as $x \rightarrow \infty$. We can therefore deduce from (5.28) that

$$
\frac{1}{x^{\gamma} L(x)} \sum_{n=s^{*}+1}^{\infty} a_{n} G_{n}(x) \rightarrow 0, \quad \text { as } \quad x \rightarrow \infty .
$$

On combining this last result with (5.19) we find that given any $\varepsilon>0$ we can choose a sufficiently large $C>0$ so that

$$
\limsup _{x \rightarrow \infty} \frac{1}{x^{\gamma} L(x)} \sum_{n=c x}^{\infty} a_{n} G_{n}(x)<\varepsilon .
$$

This result establishes (3.16) and completes our proof, since

$$
\Psi_{\eta}(x)=\sum_{n>(x / \eta)}^{\infty} a_{n} G_{n}(x) .
$$




\section{REFERENCES}

1. G. Doetsch, Handbuch der Laplace-Transformation, Vol. I, (1950) Verlag Birkhäuser, Basel.

2. B. V. Gnedenko and A. N. Kolmogorov, Limit distributions for sums of independent random variables (Translated from the Russian by K. L. Chung), (1954), Cambridge, Mass., Addison-Wesley.

3. G. H. Hardy, Divergent Series, (1949), Oxford University Press.

4. H. Hatori, Some theorems in an extended renewal theory, I, Kōdai Math. Sem. Rep., 11 (1959), 139-146.

5. — A note on a renewal theorem, Ködai Math. Sem. Rep., 12 (1960), 28-37.

6. J. Karamata, Sur un mode de croissance réguliìre des fonctions, Mathematics (Cluj), 4 (1930), 38-53.

7. M. L. Katz, The probability in the tail of a distribution, Ann. Math. Statist., 34 (1963), 312-318.

8. T. Kawata, A renewal theorem, J. Math. Soc. Japan, 8 (1956), 118-126.

9. H. Robbins and Y. S. Chow, Abstracts of short communications, (1962), International Congress of Mathematicians, Stockholm.

10. W. L. Smith, On some general renewal theorems for nonidentically distributed variables, Proceedings of the Fourth Berkeley Symposium on Mathematical Statistics and Probability, Vol. II, (1962), 467-514, Berkeley, University of California Press.

11. On functions of characteristic functions and their application to some renewal-theoretic random walk problems, (1964), to appear. 



\title{
PACIFIC JOURNAL OF MATHEMATICS
}

\author{
EDITORS
}

\author{
Robert Osserman \\ Stanford University \\ Stanford, California \\ M. G. Arsove \\ University of Washington \\ Seattle 5, Washington
}

\author{
J. Dugundji \\ University of Southern California \\ Los Angeles 7, California \\ Lowell J. Paige \\ University of California \\ Los Angeles 24, California
}

\section{ASSOCIATE EDITORS}
E. F. BECKENBACH
B. H. NEUMANN
F. WOLF
K. YOSHIDA

\section{SUPPORTING INSTITUTIONS}

\author{
UNIVERSITY OF BRITISH COLUMBIA \\ CALIFORNIA INSTITUTE OF TECHNOLOGY \\ UNIVERSITY OF CALIFORNIA \\ MONTANA STATE UNIVERSITY \\ UNIVERSITY OF NEVADA \\ NEW MEXICO STATE UNIVERSITY \\ OREGON STATE UNIVERSITY \\ UNIVERSITY OF OREGON \\ OSAKA UNIVERSITY \\ UNIVERSITY OF SOUTHERN CALIFORNIA
}

\author{
STANFORD UNIVERSITY \\ UNIVERSITY OF TOKYO \\ UNIVERSITY OF UTAH \\ WASHINGTON STATE UNIVERSITY \\ UNIVERSITY OF WASHINGTON \\ AMERICAN MATHEMATICAL SOCIETY \\ CALIFORNIA RESEARCH CORPORATION \\ SPACE TECHNOLOGY LABORATORIES \\ NAVAL ORDNANCE TEST STATION
}

Mathematical papers intended for publication in the Pacific Journal of Mathematics should by typewritten (double spaced), and on submission, must be accompanied by a separate author's résumé. Manuscripts may be sent to any one of the four editors. All other communications to the editors should be addressed to the managing editor, L. J. Paige at the University of California, Los Angeles 24, California.

50 reprints per author of each article are furnished free of charge; additional copies may be obtained at cost in multiples of 50 .

The Pacific Journal of Mathematics is published quarterly, in March, June, September, and December. Effective with Volume 13 the price per volume (4 numbers) is $\$ 18.00$; single issues, $\$ 5.00$. Special price for current issues to individual faculty members of supporting institutions and to individual members of the American Mathematical Society: $\$ 8.00$ per volume; single issues $\$ 2.50$. Back numbers are available.

Subscriptions, orders for back numbers, and changes of address should be sent to Pacific Journal of Mathematics, 103 Highland Boulevard, Berkeley 8, California.

Printed at Kokusai Bunken Insatsusha (International Academic Printing Co., Ltd.), No. 6, 2-chome, Fujimi-cho, Chiyoda-ku, Tokyo, Japan.

PUBLISHED BY PACIFIC JOURNAL OF MATHEMATICS, A NON-PROFIT CORPORATION

The Supporting Institutions listed above contribute to the cost of publication of this Journal, but they are not owners or publishers and have no responsibility for its content or policies. 


\section{Pacific Journal of Mathematics}

\section{Vol. 14, No. $2 \quad$ June, 1964}

Tom M. (Mike) Apostol and Herbert S. Zuckerman, On the functional equation $F(m n) F((m, n))=F(m) F(n) f((m, n)) \ldots \ldots \ldots \ldots \ldots \ldots \ldots \ldots \ldots \ldots \ldots$

Reinhold Baer, Irreducible groups of automorphisms of abelian groups . . . . . . . 385

Herbert Stanley Bear, Jr., An abstract potential theory with continuous kernel . . . . 407

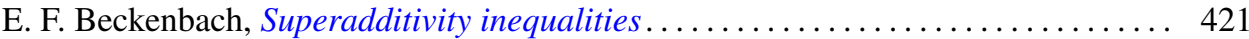

R. H. Bing, The simple connectivity of the sum of two disks . . . . . . . . . . . 439

Herbert Busemann, Length-preserving maps ...................... 457

Heron S. Collins, Characterizations of convolution semigroups of measures . . . . . . 479

Paul F. Conrad, The relationship between the radical of a lattice-ordered group and complete distributivity............................ 493

P. H. Doyle, III, A sufficient condition that an arc in $S^{n}$ be cellular . . . . . . . . . 501

Carl Clifton Faith and Yuzo Utumi, Intrinsic extensions of rings . . . . . . . . . . 505

Watson Bryan Fulks, An approximate Gauss mean value theorem . . . . . . . . . . 513

Arshag Berge Hajian, Strongly recurrent transformations . . . . . . . . . . . . . 517

Morisuke Hasumi and T. P. Srinivasan, Doubly invariant subspaces. II . . . . . . . 525

Lowell A. Hinrichs, Ivan Niven and Charles L. Vanden Eynden, Fields defined by

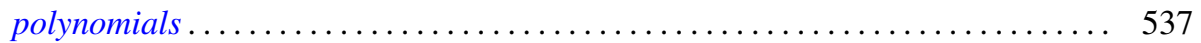

Walter Ball Laffer, I and Henry B. Mann, Decomposition of sets of group

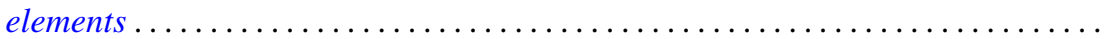

John Albert Lindberg, Jr., Algebraic extensions of commutative Banach

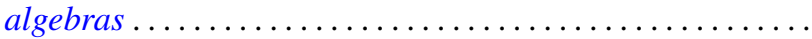

W. Ljunggren, On the Diophantine equation $C x^{2}+D=y^{n} \ldots$

M. Donald MacLaren, Atomic orthocomplemented lattices ....

Moshe Marcus, Transformations of domains in the plane and applications in the

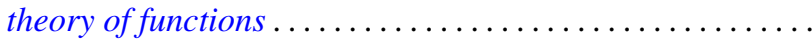

Philip Miles, $B^{*}$ algebra unit ball extremal points . .................. 627

W. F. Newns, On the difference and sum of a basic set of polynomials . . . . . . . 639

Barbara Osofsky, Rings all of whose finitely generated modules are injective ...... 645

Calvin R. Putnam, Toeplitz, matrices and invertibility of Hankel matrices . . . . . . . 651

Shoichiro Sakai, Weakly compact operators on operator algebras . . . . . . . . . 659

James E. Simpson, Nilpotency and spectral operators . . . . . . . . . . . . . 665

Walter Laws Smith, On the elementary renewal theorem for non-identically

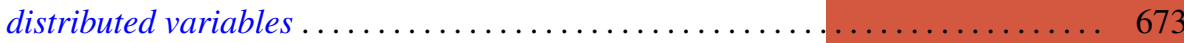

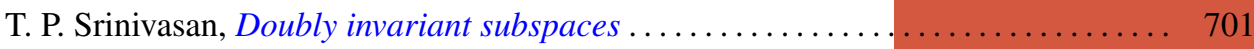

J. Roger Teller, On the extensions of lattice-ordered groups . . . . . . . . . . . . 709

Robert Charles Thompson, Unimodular group matrices with rational integers as

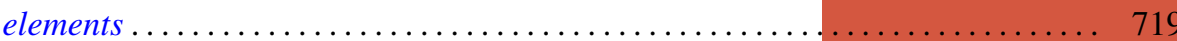

J. L. Walsh and Ambikeshwar Sharma, Least squares and interpolation in roots of unity

Charles Edward Watts, A Jordan-Hölder theorem .................... 731

Kung-Wei Yang, On some finite groups and their cohomology .............. 735

Adil Mohamed Yaqub, On the ring-logic character of certain rings ............ 741

Paul Ruel Young, A note on pseudo-creative sets and cylinders 